\title{
MÉTODO DA BISSEÇÃO PARA CÁLCULO DE RAÍZES DE FUNÇÕES POLINOMIAIS NO ENSINO BÁSICO
}

\author{
Romário Freire Santos ${ }^{1}$ \\ Queila Batista Muniz de Azevedo ${ }^{2}$
}

\section{RESUMO}

O presente trabalho apresenta uma discussão a respeito do estudo da Álgebra no Ensino Básico, mais precisamente sobre o cálculo de raízes de funções polinomiais. Nesse sentido, o objetivo é realizar estudo e apresentação de alguns métodos numéricos para a obtenção aproximada das raízes de funções, com foco principalmente no método da bisseção. No primeiro capítulo será feito levantamento bibliográfico com um apanhado histórico sobre o estudo de polinômios, com destaque dos principais estudiosos e do processo histórico de estudo a respeito do cálculo dos zeros de equações polinomiais. No segundo capítulo se realizará apresentação de algumas maneiras que o livro do Ensino Básico utiliza para cálculo da solução de equações polinomiais com grau menor que dois. Na sequência ocorrerá a apresentação do método da bisseção para cálculo de raízes exatas ou aproximadas de funções polinomiais. A conclusão do trabalho se dá com destaque da importância do aluno conhecer deferentes métodos para cálculo das raízes de funções polinomiais, de maneira que seja ativo no processo de ensino aprendizagem e não somente um reprodutor de fórmulas.

Palavras chave: Polinômios. Cálculo de raízes. Métodos numéricos. Método da bisseção.

\footnotetext{
${ }^{1}$ Mestrando em Matemática - PROFMAT (IMPA) - Universidade Estadual do Sudoeste da Bahia. Contatos: E-mail: rfsantos2012.1@gmail.com

2 Mestranda em Ciências da Educação - Facultad Interamericana de Ciências Sociales FICS - Assunção - Paraguai.

Contato: E-mail: queilabm.yen@hotmail.com
} 


\section{INTRODUÇÃO}

No Ensino Básico a introdução inicial do estudo da Álgebra se dá basicamente com o estudo de equações, com o processo de substituir valores desconhecidos por símbolos e permitir efetuar operações matemáticas de equivalência em torno de igualdades e também desigualdades.

Segundo Souza e Diniz (1996), operações em que se envolvem valores concretos, números, e que exigem um valor numérico, são permitidas a partir de conceitos e propriedades aritméticas, enquanto que a Álgebra permite expressar o que é genérico, que se pode afirmar para um conjunto de valores numéricos.

De acordo com os autores, Álgebra não é simplesmente o emprego de símbolos para substituir valores desconhecidos em determinada situação problema, se trata, portanto, da linguagem Matemática utilizada para expressar fatos genéricos.

De acordo com Dierings (2014), o desenvolvimento da álgebra com relação à resolução de problemas se deu basicamente por volta de 1500 anos antes de Cristo, problemas estes que posteriormente seriam chamados de equações polinomiais. $O$ autor ainda retrata que matemáticos egípcios e gregos estabeleceram processos práticos para encontrar as raízes de polinômios de primeiro grau relacionados com situações do cotidiano.

Mais adiante, por volta de 400 anos antes de Cristo, o matemático Diofanto de Alexandria apresentou as primeiras maneiras algébricas para resolução de equações do segundo grau. Segundo Teles (2004) e Dierings (2014), Diofanto foi o responsável pela mudança da maneira de registrar as expressões, onde que se deixou de registrar totalmente em palavras e passou a usar também abreviações.

$\mathrm{Na}$ Grécia, por volta de 300 a.C., Euclides de Alexandria, professor do Museu de Alexandria, publicou 13 livros em sua obra Elementos, dos quais dois são dedicados especificamente à Álgebra. Euclides preocupava em associar os valores desconhecidos às figuras geométricas, com a intenção de perceber as relações existentes. (TELES, 2004).

É importante destacar que os Axiomas de Euclides favoreceram para a sistematização do processo de resolução de equações polinomiais de primeiro grau, principalmente os seguintes axiomas: 
i) Se duas coisas são iguais a uma terceira, então são iguais entre si.

ii) Se iguais são somados (ou subtraídos) a iguais, os resultados permanecem iguais.

iii) O todo é maior que a parte.

Estes axiomas configuram a relação de equivalência existente no processo algébrico de resolução das equações de primeiro grau.

No século VIII depois de Cristo o matemático Mohammed ibn-Musa alKhowarizmi introduziu novos símbolos nas expressões algébricas, mas, assim como outros matemáticos da época, não foi capaz de reduzir as expressões somente ao uso de símbolos (DIERINGS, 2014). O autor destaca que al-Khowarizmi e Bháskara empregavam praticamente o mesmo procedimento para resolução das equações polinomiais do segundo grau, com abordagem da noção de complemento de quadrados.

De acordo com Dierings (2014), o matemático italiano Niccoló Fontana Tartaglia (1500-1557) desenvolveu o método de resolução de equações polinomiais do terceiro grau do tipo $\mathbf{x}^{3}+\mathbf{p x}+\mathbf{q}=\mathbf{0}$, método que foi publicado por Gerolamo Cardano na Ars Magma. No entanto, Cardano relata que o professor Scipione Del Ferro já havia desenvolvido tal método. Posteriormente Cardano desenvolve uma fórmula para resolução de equações polinomiais do quarto grau, a partir de um processo de redução para terceiro grau.

Pode se destacar que o matemático francês, François Viète (1540-1603), foi responsável pela introdução do uso de letras para representar valores desconhecidos e também dos símbolos nas operações, da maneira como são utilizados até hoje. Na sequência, como descreve Teles (2004, p.8), "A passagem para a Álgebra simbólica foi completada pelo grande matemático e filósofo francês René Descartes (1596-1650), que aperfeiçoou a Álgebra de Viète, criando a notação que usamos até hoje para expoentes". Assim, o objeto de estudo da Matemática deixa de ser somente situações do cotidiano envolvendo problemas numéricos, mas as próprias expressões algébricas.

Descartes e Pierre de Fermat criaram, em tempo praticamente simultâneo, a Geometria Analítica. Dentre as áreas de estudo de Fermat, o estudo de funções contribuiu com o processo para traçar retas tangentes à curvas e também com o cálculo de máximos e mínimos. Enquanto isso, Descartes desenvolve um processo 
algébrico para traçar retas tangentes às curvas e na sequência contribui para a definição e aceitação de raízes quadrada de números negativos como solução de equações. (DIERINGS, 2014).

Ainda com relação ao cálculo algébrico de raízes de equações polinomiais, Dierings (2014) relata que muito tempo e energia foram utilizados para encontrar raízes de polinômios que têm grau maior ou igual a 5. O autor destaca que em 1799, Carl Friedrich Gauss, com motivação de Joseph Louis Lagrange, prova o Teorema Fundamental da Álgebra em que enuncia que toda equação algébrica com grau maior ou igual a 1 admite ao menos uma raiz complexa.

No início do século XIX, Paolo Ruffini, que foi, além de matemático, médico italiano, provou que não existe fórmula específica para resolver equações polinomiais do quinto grau. Na sequência, o matemático norueguês Niels Henrik Abel mostrou que não é possível resolver equações com grau superior a quatro através dos seus radicais. Afirmação esta que se fundamenta também nas pesquisas e estudos do jovem matemático francês Évariste Galois (1811-1832).

Diversos outros matemáticos continuaram a pesquisar sobre métodos de resolução de equações, e este processo de investigação contribuiu veementemente para o desenvolvimento da Álgebra. O autor Dierings (2014) relata que métodos numéricos (iterativos) tiveram ênfase neste processo de desenvolvimento da Álgebra, além dos pesquisadores que já aparecem no texto, pode se citar, dentre diversos outros, "Daniel Bernoulli, Karl Heinrich Graeffe e Jean Batiptiste Joseph Fourier e Évariste Galois" (DIERINGS, 2014, p. 16).

\subsection{ESTUDO DE EQUAÇÕES POLINÔMIAIS NO ENSINO BÁSICO}

O primeiro contato dos alunos com as equações polinomiais do primeiro grau (ou algébricas) ocorre no oitavo ano (antiga $7^{\underline{a}}$ série), onde se estuda a respeito da formação dessas equações e na sequência se tem a resolução, o cálculo do zero da equação. Posteriormente, no nono ano (antiga $8^{\underline{a}}$ série), estuda sobre as equações de primeiro e de segundo graus, e suas funções associadas.

O estudo sobre as equações polinomiais do primeiro e do segundo graus somente pelo processo algébrico e com o cálculo da raiz de maneira mecânica pode 
não ser suficiente no processo de ensino aprendizagem. Apresentar o conteúdo, expor enunciados e exemplos, técnicas de resolução, e na sequência solicitar que se responda listas de exercícios semelhantes, pode não ser eficaz para instigar a construção do conhecimento crítico a respeito das equações polinomiais e desenvolvimento do conceito, que torna extremamente abstrato e sem o devido significado para o aprendiz. (NASCIMENTO, 2015).

Como relata Nascimento (2015), frequentemente o estudo de equações algébricas ocorre de maneira em que se calcula a raiz por um processo mecânico, sem fazer uso da didática para ressaltar e explicar sobre as propriedades algébricas envolvidas. Dessa maneira, não instiga o aluno a questionar e indagar sobre a outros processos de resolução, como o de testar valores.

Ainda segundo Nascimento (2015), estimular o aluno a pensar em outras maneiras de encontrar as soluções das equações e os zeros das funções, pode contribuir para que este entenda sobre as propriedades envolvidas no processo de equivalência das igualdades e sobre o que significa a solução da equação e o zero da função.

Os Parâmetros Curriculares Nacionais (PCNs), que têm como um de seus eixos a Álgebra, destacam e orientam quanto à importância do estudo sobre funções, ao enfatizar que:

O estudo das funções permite ao aluno adquirir a linguagem algébrica como a linguagem das ciências, necessária para expressar a relação entre grandezas e modelar situações-problema, construindo modelos descritivos de fenômenos e permitindo várias conexões dentro e fora da própria matemática (BRASIL, 2002, p. 121).

Dessa maneira, é de fundamental importância o conhecimento a respeito de métodos iterativos que permitam o cálculo de raízes de funções polinomiais, já que frequentemente se torna necessário descobrir valor ou valores que tornam a função nula. Isto corrobora com as palavras de Ruggiero e Lopes ao descreverem que "nas mais diversas áreas das ciências exatas ocorrem, frequentemente, situações que envolvem a resolução de uma equação do tipo $f(x)=0$ " (RUGGIERO e LOPES, 1997, p. 27). 


\section{POLINÔMIOS}

Definição: Seja um número $\mathrm{n} \in \mathbb{N}$ e $a_{n}, a_{n-1}, a_{n-2}, \ldots, a_{2}, a_{1}, a_{0} \in \mathrm{C}$, onde $C$ é o conjunto dos números complexos, denomina-se função polinomial ou simplesmente polinômio a expressão algébrica da forma:

$\mathbf{p}(\mathbf{x})=a_{n} x^{n}+a_{n-1} x^{n-1}+a_{n-2} x^{n-2}+\cdots+a_{2} x^{2}+a_{1} x^{1}+a_{0}$,

com C $\rightarrow$ C. Onde:

- $a_{n}, a_{n-1}, a_{n-2}, \ldots, a_{2}, a_{1}, a_{0}$ são denominados coeficientes

- $x \in C$ a variável

Por exemplo:

A função polinomial dada por $p(x)=2 x^{3}+x^{2}+3 x+4$, se tem a variável $\mathbf{x}$ e os coeficientes $a_{3}=2, a_{2}=1, a_{1}=3, a_{0}=4$.

Denota como grau do polinômio o maior expoente da variável $\mathbf{x}$, o exemplo anterior é um polinômio de grau 3.

Os estudantes do Ensino Médio estudam incialmente a funções polinomiais de primeiro grau e segundo grau, sendo chamadas respectivamente de funções afins e funções quadráticas. No estudo dessas funções observam os valores da função para diferentes valores do domínio, as construções dos diferentes gráficos, os zeros da função com métodos específicos, crescimento e decrescimento. Torna interessante o uso de softwares, como o Geogebra, para ilustrar os diferentes comportamentos das funções polinomiais.

\section{Exemplos:}

- Seja a função afim, $\mathbf{p}(\mathbf{x})=\mathbf{2 x}-\mathbf{3}$.

Ao atribuir valores para $x$ e calcular as respectivas imagens $(p(x))$, pode se construir o gráfico, analisar que a função é crescente, o ponto que intersecta o eixo das ordenadas $(y=-3)$ e perceber que valor de $x$ que torna a função nula, o valor em que intersecta o eixo das abscissas, $x=3 / 2$, é justamente o que se define como zero da função.

- Função quadrática, exemplo $p(x)=x^{2}-9$. 
Construir o gráfico também no caderno, atribuindo valores para $\mathrm{x}$, e calcular os zeros da função, $-3 \mathrm{e}+3$, o ponto que intersecta o eixo das ordenadas $(y), y=-9$, o vértice da função no ponto $(0,-9)$.

Nas funções de terceiro e quarto graus também pode se observar e analisar a partir do gráfico e de cálculos numéricos, juntamente com estudantes, os intervalos de crescimento e decrescimento das funções, pontos que intersecta o eixo $x$, ou seja quando $p(x)=0$.

- A função polinomial de terceiro grau, $p(x)=x^{3}+4 x^{2}+x-6$, com seguinte gráfico:

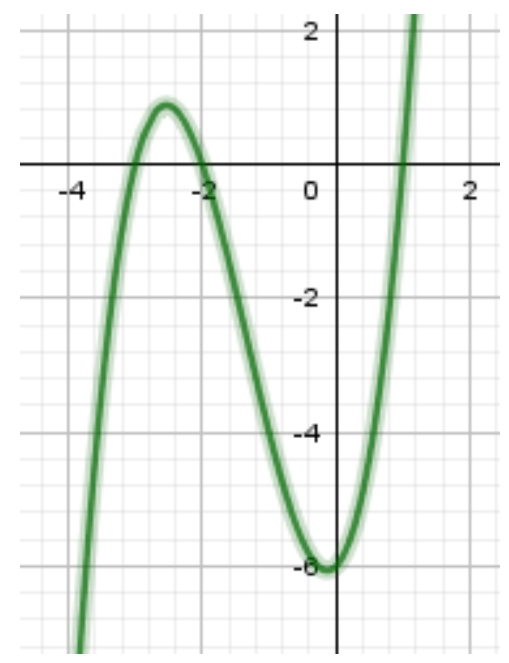

Figura 1

A função polinomial de quarto grau $p(x)=x^{4}+x^{3}-7 x^{2}-x+6$ 


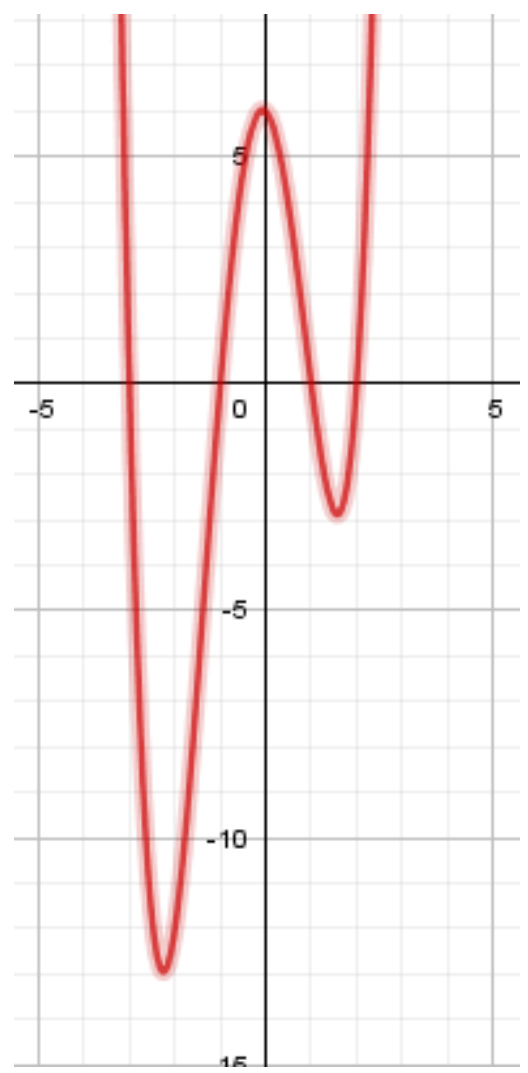

Figura 2

O estudo das propriedades sobre polinômios em consonância com análise de gráfico pode contribuir para a construção do conhecimento acerca do comportamento de funções polinomiais.

\subsection{RAIZ DE FUNÇÃO POLINOMIAL}

Definição: Seja $p(x)$ um polinômio sobre $C$, definida de $p: C \rightarrow C$. A raiz desse polinômio é o ponto $\alpha \in C$, se $p(\alpha)=0$.

Exemplo:

$$
p(x)=x+2
$$

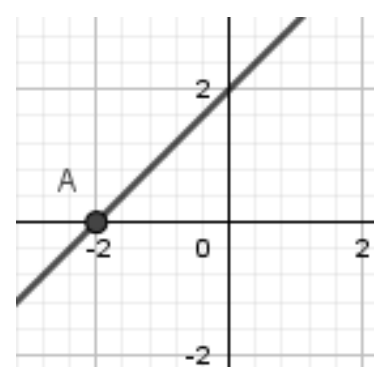

Figura 3 
No gráfico (figura 3) percebemos que o ponto que o gráfico intersecta o eixo das abscissas é em $(-2,0)$. Nesse ponto a função $p(x)=0$, portanto -2 é raiz da função.

Alguns métodos são utilizados na obtenção de raízes de funções Polinomiais.

Nas funções afins e quadráticas, esses métodos são específicos e possuem facilidade para encontrar o valor de $\mathrm{x}$ desejado.

Funções Afins

A função afim, representada por $p(x)=a_{1} x^{1}+a_{0}$, ou simplesmente $p(x)=a x+b$, com $\mathrm{a}, \mathrm{b} \in \mathrm{C}$ e $a \neq 0$. Igualando $\mathrm{p}(\mathrm{x})$ a zero, ficará fácil de determinar a raiz da equação.

Sendo:

$$
a x+b=0
$$

Sendo a e b números reais, a raiz da equação será encontrada por -b/a.

Exemplo: $p(x)=2 x-8$, temos os coeficientes $\mathrm{a}=2$ e $\mathrm{b}=-8$, então $-\frac{b}{a}=-\left(-\frac{8}{2}\right)=$ $-(-4)=4$.

Então a raiz da função $p(x)=2 x-8$ é igual a 4 .

\section{Funções Quadráticas}

As funções Quadráticas ou de segundo grau são da forma polinomial $p(x)=a_{2} x^{2}+a_{1} x^{1}+a_{0}$, também representada por $p(x)=a x^{2}+b x+c$, com $a, b$, c $\in \mathrm{R}$ e $a \neq 0$.

Uma fórmula bastante conhecida para resolução da função Quadrática, é a fórmula de Bhaskara (1.114-1.185).

Desenvolvendo a fórmula de Bhaskara:

$$
p(x)=a x^{2}+b x+c
$$

Igualando $\mathrm{p}(\mathrm{x})=0$

$$
\begin{gathered}
a x^{2}+b x+c=0 \rightarrow a\left(x^{2}+\frac{b}{a} x+\frac{c}{a}\right)=0 \\
\Rightarrow x^{2}+\frac{b}{a} x=-\frac{c}{a} \rightarrow x^{2}+\frac{b}{a} x+\frac{b^{2}}{4 a^{2}}=-\frac{c}{a}+\frac{b^{2}}{4 a^{2}} \\
\Rightarrow\left(x+\frac{b}{2 a}\right)^{2}=\frac{b^{2}-4 a c}{4 a^{2}} \rightarrow x+\frac{b}{2 a}= \pm \frac{\sqrt{b^{2}-4 a c}}{2 a}
\end{gathered}
$$




$$
\Rightarrow x=-\frac{b}{2 a} \pm \frac{\sqrt{b^{2}-4 a c}}{2 a}
$$

O radicando $b^{2}-4 a c$, também é chamado de $\Delta$ (delta). Assim temos:

$$
x=\frac{-b \pm \sqrt{\Delta}}{2 a}
$$

Assim as raízes da função quadrática serão $x_{1}=\frac{-b+\sqrt{\Delta}}{2 a}$ e $x_{2}=\frac{-b-\sqrt{\Delta}}{2 a}$, e teremos:

Quando $\Delta=0$, há duas raízes reais idênticas

Quando $\Delta<0$, há duas raízes complexas, ou seja não existe raízes reais

Quando $\Delta>0$, há duas raízes reais e distintas

Exemplo: $p(x)=3 x^{2}-4 x+1$, Temos os coeficientes, $\mathrm{a}=3, b=-4$ e $c=1$

$$
\text { Sendo } \Delta=b^{2}-4 a c \rightarrow \Delta=(-4)^{2}-4.3 .1=4
$$

E as raízes da função serão:

$$
\begin{aligned}
& x=\frac{-(-4) \pm \sqrt{4}}{2.3} \\
& x_{1}=\frac{4+2}{6}=1 \\
& x_{2}=\frac{4-2}{6}=\frac{1}{3}
\end{aligned}
$$

Com seguinte gráfico:

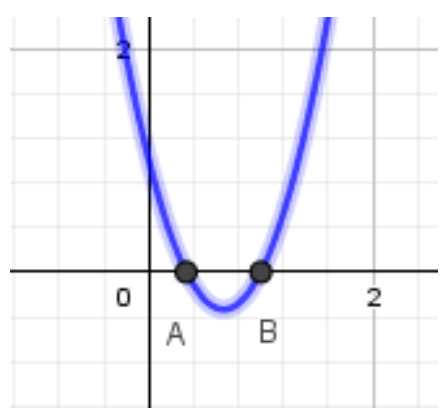

Figura 4

Percebemos que o gráfico corta o eixo das abscissas em 1 e $\frac{1}{3}$, portanto essas são as raízes das função quadrática.

Dessa maneira, a fórmula de Bhaskara pode ser um método eficiente para calcular as raízes da função quadrática. 


\subsection{MÉtodos DE ITERAÇÃo NO CÁlCULO RAízeS DE FUNÇÕES POLINOMIAIS}

No decorrer deste trabalho se apresentou métodos específicos e de cálculos simples para encontrar raízes de funções afins e quadráticas. Já para encontrar raízes de polinômios de grau 3 e 4, as fórmulas tornam-se mais complicadas. Diante disso, surge a indagação: como calcular as raízes de funções polinomiais com grau maior que 2, de maneira rápida e segura.

Para isto existem métodos iterativos que podem ajudar a encontrar raízes de funções diversas. Dentre esses métodos, pode se destacar os métodos da Bissecção, Método da Posição Falsa, Método do Ponto Fixo, Método de NewtonRaphson e o Método da Secante

Em sala de aula, no ensino médio, pode se utilizar alguns desses métodos de fácil entendimento e de cálculo simples. Além disso, o entendimento desses métodos, contribuirá para o estudante compreender os gráficos dos diversos polinômios e perceber as características dessas funções

No cálculo de Raízes de funções, temos duas fases importantes:

1. Isolamento de raízes, consiste em encontrar um intervalo que contém pelo menos uma raiz.

2. Refinamento do intervalo, consiste, de aproximar a raiz procurada, dentro do intervalo já definida na fase 1 .

\section{ISOLAMENTO}

Nessa fase, se analisa a função $p$, com determinados valores para $x$, e o resultado em $\mathrm{p}(\mathrm{x})$.

Pode se imaginar, que com um $p\left(x_{1}\right)<0 e$ um $p\left(x_{2}\right)>0$, com $x_{1}<x_{2}$, existirá

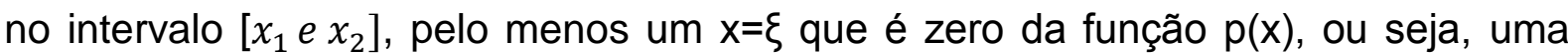
raiz da função.

Graficamente: 


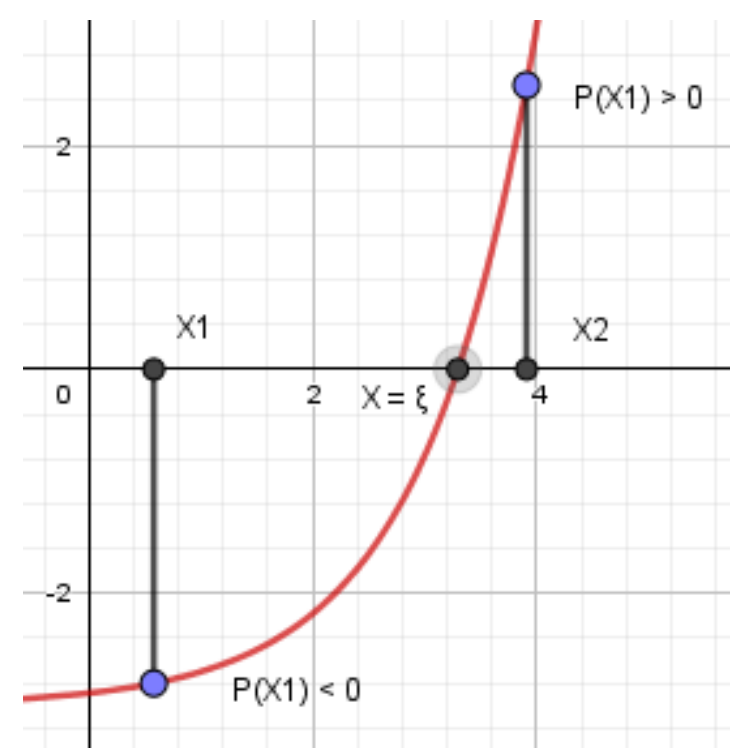

Figura 5

Com estudantes do ensino médio, pode se abordar valores sequenciais para $x$ e verificar os resultados em $p(x)$, sempre que houver mudança de sinal no resultado, haverá pelo menos uma raiz naquele intervalo. Assim estará definido um intervalo que contém pelo menos uma raiz. É interessante instigar a investigação, de maneira que $\mathrm{o}$ aluno analise a mudança $\mathrm{em} \mathrm{p}(\mathrm{x})$ para diferentes valores de $\mathrm{x}$.

Por exemplo:

A função $p(x)=x^{5}-2 x^{4}-4 x^{3}+x+1$, atribuímos valores para $\mathrm{x}$.

\begin{tabular}{|l|l|}
\hline $\mathbf{x}$ & $\mathbf{p}(\mathbf{x})$ \\
\hline-2 & -97 \\
\hline-1 & 1 \\
\hline 0 & 1 \\
\hline 1 & -3 \\
\hline 2 & -29 \\
\hline
\end{tabular}

Verifica-se que houve mudança de sinal em $p(x)$ no intervalo $[-2,-1]$ e no intervalo $[0,1]$, portanto é seguro afirmar que em cada um desses intervalos existe pelo menos uma raiz da função.

A informação da existência das raízes nos intervalos analisados pode ser analisada a partir do gráfico da função $\mathrm{p}(\mathrm{x})$, esboçado a partir do software Geogebra. Este software é uma ferramenta que o professor ao apresentar para os alunos pode instigar o uso, de maneira que o comecem a testar e conhecer suas ferramentas, para que assim possam a usar com foco também em conteúdos da Matemática. 


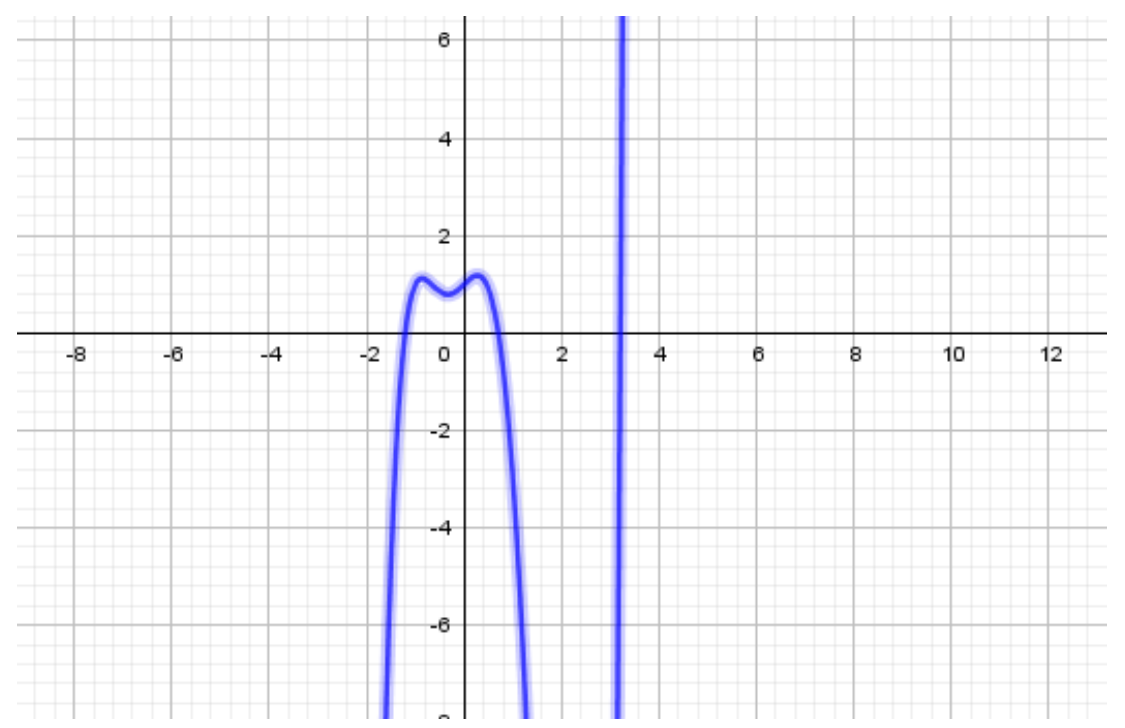

Figura 6

\section{O REFINAMENTO}

Depois de selecionar um intervalo que contem a raiz, o próximo passo é o refinamento, ou seja, reduzir o intervalo de tal maneira a aproximar do zero da função de acordo com a precisão desejada.

Existem diversos métodos numéricos para refinar a raiz contida no intervalo. Esses métodos recebem o nome de iteração, por se tratar de cálculos realizados em sequência, sendo utilizado o resultado do cálculo anterior até alcançar a aproximação desejada.

\subsubsection{MÉTODO DA BISSEÇÃO}

Dentre os métodos numéricos, temos a Bissecção, que é simples e interessante para as aulas no ensino Médio. Consiste em divisões sucessivas, para reduzir a amplitude do intervalo, até atingir a precisão buscada.

Tendo a função $\mathrm{p}(\mathrm{x}), \operatorname{com} p(a) \cdot p(b)<0$, temos o intervalo [a,b] que consta a raiz. Faremos a divisões sucessivas, até encontrar a aproximação desejada ou seja $(b-a)<\varepsilon$, sendo $\varepsilon$ o erro máximo. 
As iterações são da seguinte forma:

$$
x_{0}=\frac{a+b}{2}, \operatorname{com} p(a)<0 \text { e } p(b)>0 \text {, se } p\left(x_{0}\right)>0 \text {, temos } \xi €\left[a, x_{0}\right]
$$

Fazendo $b_{1}=x_{0}$ e $a=a_{1}$ nesse novo intervalo fazemos o mesmo;

$$
x_{1}=\frac{a_{1}+b_{1}}{2}, \operatorname{com} p\left(a_{1}\right)<0 \text { e } p\left(b_{1}\right)>0 \text {, se } p\left(x_{1}\right)<0 \text {, temos } \xi €\left[x_{1}, b_{1}\right]
$$

Fazendo $x_{1}=a_{2}$ e $b_{1}=b_{2}$, continuamos as iterações;

$$
x_{2}=\frac{a_{2}+b_{2}}{2} \text {, com } p\left(a_{2}\right)<0 \text { e } p\left(b_{2}\right)>0 \text {, se } p\left(x_{2}\right)<0 \text {, temos } \xi \in\left[x_{2}, b_{2}\right]
$$

E dessa forma continuemos as iterações, sempre escolhendo o intervalo com $p(a) \cdot p(b)<0$, até alcançar a aproximação desejada onde $(b-a)<\varepsilon$.

Com o método da bissecção, o estudante poderá encontrar as raízes de funções polinomiais, assim como de outras funções, como as trigonométricas.

Exemplo: Calcular a raiz de $p(x)=x^{5}-2 x^{4}-4 x^{3}+x+1$.

Já foi visto anteriormente que no intervalo $[0,1]$ possui pelo menos uma raiz, portanto, fazendo as iterações, temos:

\begin{tabular}{|r|r|r|r|r|r|r|}
\hline$a$ & \multicolumn{1}{|c|}{$b$} & $(a+b) / 2$ & $p(a)$ & $p(b)$ & $p\left(\frac{a+b}{2}\right)$ & $(a-b)$ \\
\hline 0 & 1 & 0,5 & 1 & -3 & 0,90625 & 1 \\
\hline 0,5 & 1 & 0,75 & 0,90625 & -3 & $-0,333$ & 0,5 \\
\hline 0,5 & 0,75 & 0,625 & 0,90625 & $-0,333$ & 0,4386 & 0,25 \\
\hline 0,625 & 0,75 & 0,6875 & 0,4386 & $-0,333$ & 0,0944 & 0,125 \\
\hline 0,6875 & 0,75 & 0,71875 & 0,0944 & $-0,333$ & $-0,1084$ & 0,0625 \\
\hline 0,6875 & 0,71875 & 0,703125 & 0,0944 & $-0,1084$ & $-0,0043$ & 0,03125 \\
\hline 0,6875 & 0,703125 & 0,6953125 & 0,0944 & $-0,0043$ & 0,0457 & 0,01562 \\
\hline 0,695312 & 0,703125 & 0,6992185 & 0,0457 & $-0,0043$ & 0,0208 & 0,00781 \\
\hline 0,6992185 & 0,703125 & 0,7011717 & 0,0209 & $-0,0043$ & 0,008 & 0,0039 \\
\hline
\end{tabular}

No gráfico abaixo é possível acompanhar algumas das iterações realizadas. Os pontos A, B, C, D, E, F, H representam, nessa ordem, a aproximação para a raiz da função, que é o ponto de interseção do gráfico (verde) da função com o eixo das abcissas. 


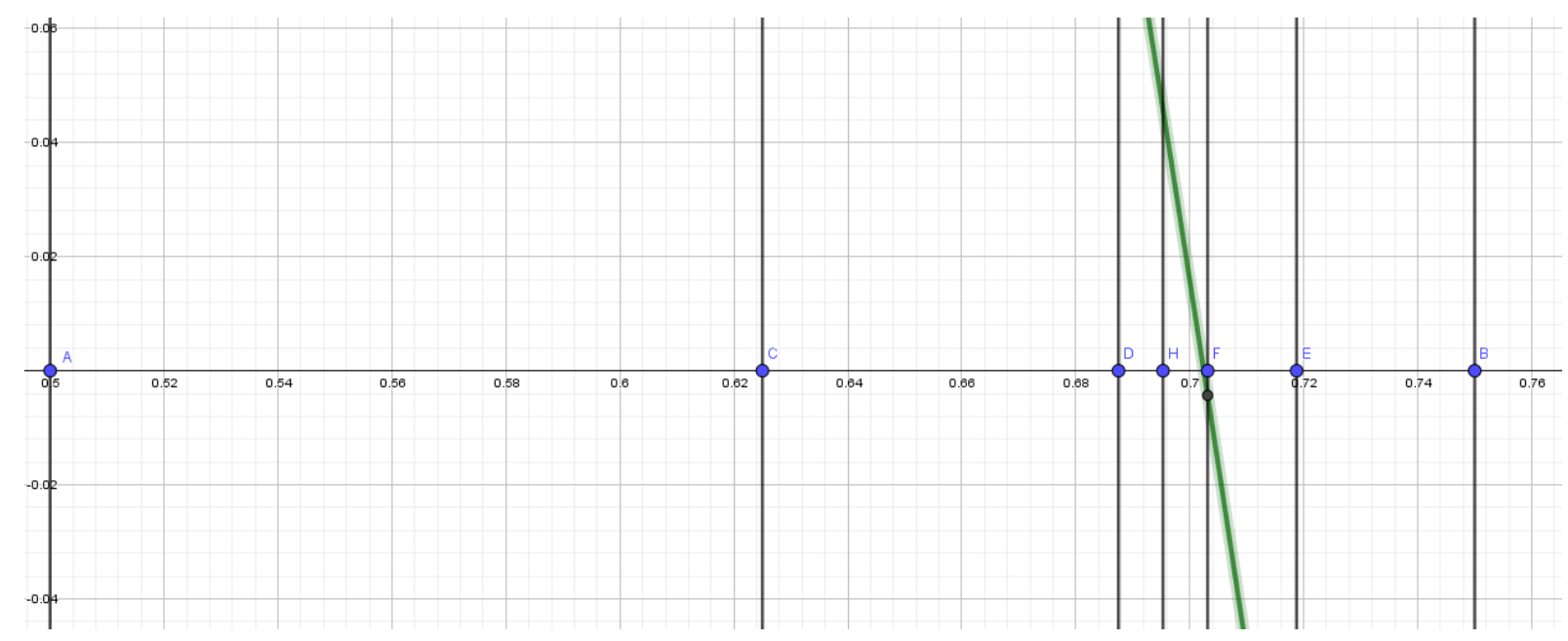

Figura 6

Obtém que a raiz estará no intervalo de [0,1875; 0,25], caso seja necessário uma aproximação com maior precisão para a raiz, basta continuar as iterações, de maneira que a raiz é o $\overline{\mathbf{x}}=\frac{\mathbf{a}+\mathbf{b}}{2}$, tal que $\boldsymbol{f}(\overline{\mathbf{x}})$ estará próximo de zero com a precisão que se deseja. A raiz encontrada pelas iterações na tabela anterior foi 0,008 , ou seja, com precisão de duas casas decimais.

Esse método não utiliza cálculos sofisticados e, portanto, pode ser utilizado com estudantes do ensino médio, como uma outra maneira para calcular raízes, sem ter que decorar fórmulas obtidas a partir dos coeficientes do polinômio.

Dada uma função, inicialmente descobrirão um intervalo que existe a raiz da função e posteriormente usarão o método da bissecção para refinar a raiz através de iterações.

Como instrumento poderão utilizar o Excel para construir as planilhas mostradas e construir os gráficos de dispersão.

\subsection{1 (I) QUANTIDADE DE ITERAÇÕES}

Para estimar a quantidade de iterações mínimas necessárias para alcançar o erro $\varepsilon$, em que $(b-a)<\varepsilon$. Temos:

$b_{k}-a_{k}=\left(b_{0}-a_{0}\right) / 2^{k}$

Para obter $\boldsymbol{b}_{\boldsymbol{k}}-\boldsymbol{a}_{\boldsymbol{k}}<\boldsymbol{\varepsilon}$, ou seja: 


$$
\begin{gathered}
\left(b_{0}-a_{0}\right) / 2^{k}<\varepsilon \\
\Rightarrow\left(b_{0}-a_{0}\right) / 2^{k}<\varepsilon \\
\Rightarrow\left(b_{0}-a_{0}\right) / \varepsilon<2^{k} \\
\Rightarrow k \cdot \log 2>\log (b-a)-\log \varepsilon \\
\Rightarrow k>\frac{(\log (b-a)-\log \varepsilon)}{\log 2}
\end{gathered}
$$

Dessa maneira se calcula a quantidade mínima de iterações necessárias k, a partir de um intervalo $[a, b]$ e um erro definido $\varepsilon$.

Com isso o método da Bissecção, torna-se um instrumento para estudantes do ensino médio encontrar raízes de funções polinomiais.

\subsection{1 (II) QUANTIDADE DE RAÍZES DE FUNÇÃO POLINOMIAL}

Quantidade de raízes pode ser definida através da Regra de Sinal de Descartes, "Dado um polinômio com coeficientes reais, o número de zeros reais, $p$, desse polinômio não excede o número $v$ de variações de sinal dos coeficientes. Ainda mais $v-p$ é inteiro, par, não negativo".

Como por exemplo:

$$
p(x)=x^{5}-2 x^{4}-4 x^{3}+x+1
$$

Ocorrem as seguintes variações dos sinais das incógnitas, +--++ , portanto são duas variações.

Então $v=2$, assim, deve ser $v-p=0, \operatorname{com} p=2$, ou pode ser $v-p=$ $2, \operatorname{com} p=0$, sendo $p$ o número de raízes.

Para determinar as raízes negativas, faça, $p(-x)$ e use a mesma regra das raízes positivas:

Exemplo:

$$
p(x)=x^{5}-2 x^{4}-4 x^{3}+x+1
$$

Fazendo $\mathrm{p}(-\mathrm{x})$, temos $p(x)=-x^{5}-2 x^{4}+4 x^{3}-x+1$ 
Dessa forma temos, $v=3 \operatorname{com} v-p=0$ temos $p=3$, com $v-p=2$, temos $p=1$

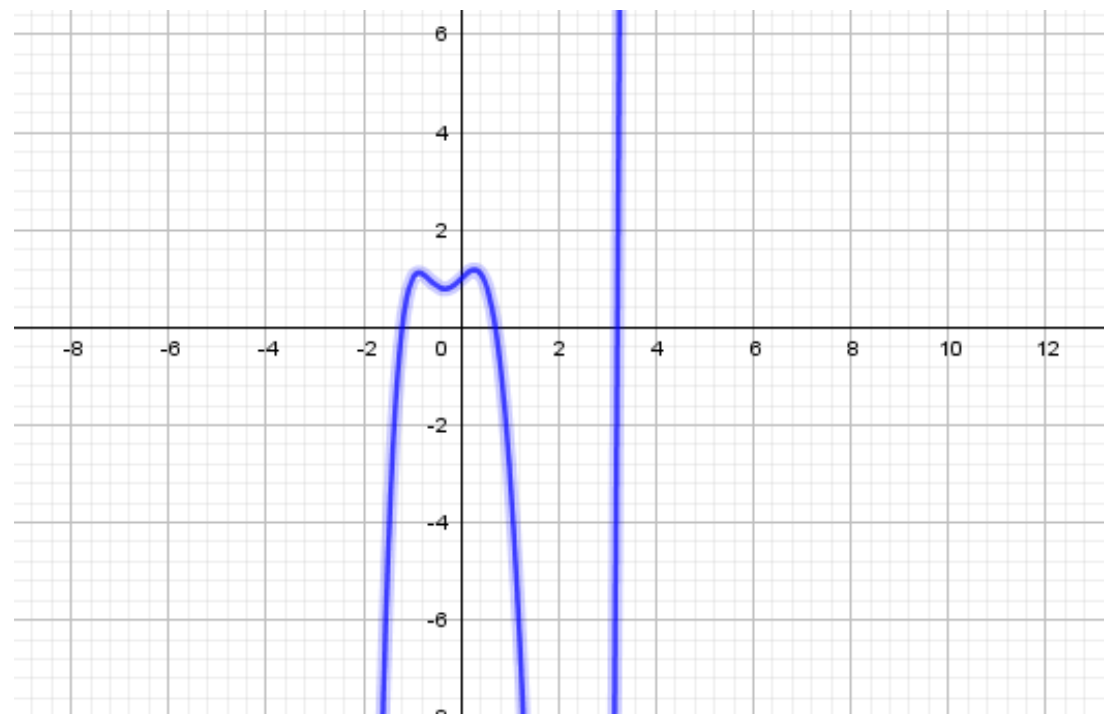

Figura 7

Dessa forma se calcula a quantidade de raízes que pode encontrar através dos métodos mostrados.

\section{CONSIDERAÇÕES FINAIS}

Uma das finalidades deste trabalho foi apresentar um breve resumo do estudo e abordagem da Matemática no Ensino Básico, com foco principalmente na Álgebra, no estudo de equações e funções polinomiais no Ensino Básico. Como já foi apresentado no decorrer do trabalho, faz parte da construção do conceito de polinômios entender o que significa a raiz de um polinômio e sobretudo saber calcular ou evidenciar estas raízes.

No entanto, como destaca Nascimento (2015), o que ocorre em sala de aula na maioria dos casos é um estudo limitado acerca das operações e propriedade envolvendo polinômios, de maneira que aluno se limita a apenas decorar uma série de fórmulas matemáticas. E assim o estudo de raízes pode se limitar a somente encontrar um valor ou valores de " $x$ ", sem, no entanto, entender o processo 
envolvido e o significado das raízes para uma função polinomial, seja o significado algébrico, contextual ou geométrico, dentre outros.

Dessa maneira é de fundamental importância que o aluno participe ativamente do processo de cálculo das raízes das funções polinomiais, o que pode instigar o aluno a imaginar outras situações diferentes daquelas apresentadas, tornando assim o conhecimento mais significativo para o aluno, uma vez que não se limita a um processo mecânico de repetição comandos (NASCIMENTO, 2015).

Contudo, o objetivo do trabalho foi destacar a respeito da existência de diversos métodos numéricos para cálculo de raízes de funções, de maneira que o professor pode abordar didaticamente no Ensino Básico. O foco principal foi sobre o método da bisseção, em que o aluno não se limita a apenas conhecer expressões algébricas para resolução de equações polinomiais de primeiro e segundo graus, mas participa do processo de aproximação da(s) raiz(es) de funções polinomiais. Assim foi feita uma abordagem do método da bisseção no cálculo de zeros de funções polinomiais de terceiro grau, entretanto o método pode ser aplicado para polinômios de qualquer grau e também em outras funções que não sejam polinomiais. 


\section{REFERÊNCIAS}

BRASIL, Secretaria de Educação Média e Tecnológica. PCN+ Ensino Médio: Orientações Educacionais Complementares aos Parâmetros Curriculares Nacionais. Linguagens, Códigos e suas Tecnologias. Brasília: Ministério da Educaçã/Secretaria de Educação Média e Tecnológica, 2002.

DIERINGS, André Ricardo. Ensino de Polinômios no Ensino Médio - Uma nova abordagem / André Ricardo Dierings.-2014. 70 p.; 30cm. Dissertação (Mestrado em Matemática - Profmat) - Universidade Federal de Santa Maria.

NASCIMENTO, Demilson Antonio do et al. Métodos para Encontrar Raízes Exatas e Aproximadas de Funções Polinomiais até o 4 Grau. 2015.

RUGGIERO, Márcia A. Gomes; LOPES, Vera Lúcia da Rocha. Cálculo numérico: aspectos teóricos e computacionais. Makron Books do Brasil, 1997.

SOUZA, Eliane Reame e DINIZ, Maria Ignez de S. Vieira. Álgebra: das Variáveis às Equações e Funções. São Paulo: IME-USP, 1996.

TELES, R. A. de M. A. Aritmética e a álgebra na matemática escolar. Educação Matemática em Revista, São Paulo: SBEM, ano, v. 11, p. 8-15, 2004. 